\title{
Microcirculação na sepse: aspectos fisiopatológicos e diagnósticos
}

\author{
Microcirculation in sepsis: \\ pathophysiological and diagnostic features
}

\author{
Marcos L. Miranda* \\ Daniella M. L. Caixeta \\ Eliete Bouskela
}

\section{Resumo}

Pela sua alta incidência, morbidade, mortalidade e custos ao sistema de saúde, a sepse se destaca entre as diversas indicações de internação em unidade de terapia intensiva. A disfunção da microcirculação tem papel central na gênese e manutenção da síndrome séptica, sendo um marco fisiopatológico desta síndrome. De fato, pode-se dizer que a sepse é uma desordem da microcirculação, sendo este o primeiro órgão a falhar durante a progressão da sepse para a síndrome de disfunção de múltiplos órgãos e morte. Sabe-se atualmente que, a despeito de um tratamento adequado da macro-hemodinâmica, existe pouca correlação entre a melhora hemodinâmica sistêmica e a correção das anormalidades microcirculatórias, sendo a presença destas anormalidades mais comum entre os pacientes não sobreviventes que naqueles sobreviventes. A razão para esta disparidade entre a macro e a micro-hemodinâmica é a presença de shunts funcionais na microcirculação que "escondem" a disfunção microcirculatória da circulação sis-

Revista HUPE, Rio de Janeiro, 2013;12(3):21-30 doi:10.12957/rhupe.2013.7527 têmica. Por não avaliarem os eventos intrínsecos que ocorrem na microcirculação, os sistemas de monitorização hemodinâmica convencionais disponíveis na prática clínica falham em detectar estas anormalidades e a resposta aos tratamentos. Este conhecimento aponta para uma grande necessidade no diagnóstico e tratamento da sepse: a avaliação diagnóstica da disfunção da microcirculação. Felizmente, existem alguns métodos diagnósticos direcionados especificamente para a avaliação das alterações microcirculatórias. Estes métodos podem ser divididos em biomarcadores, técnicas de videomicroscopia, técnicas para a avaliação da oxigenação tissular e técnicas para avaliação da microcirculação a partir da pressão parcial de gás carbônico. Visando um melhor entendimento dos processos fisiopatológicos envolvidos na disfunção microcirculatória secundária à sepse e dos meios diagnósticos atualmente disponíveis, alguns conceitos fundamentais são apresentados.

Descritores: Microcirculação; Sepse; Monitorização fisiológica; Terapia intensiva.

"Endereço para correspondência: Centro de Tratamento Intensivo Geral, HUPE, UERJ. Boulevard 28 de setembro, 77 Rio de Janeiro, RJ, Brasil. CEP: 20551-030. E-mail: marcoslm@centroin.com.br 


\section{Abstract}

Due to its high incidence, morbidity, mortality and cost to the healthcare system, sepsis stands out among the many indications for intensive care unit admission. The microcirculatory dysfunction plays a central role in the genesis and maintenance of the septic syndrome, being a pathophysiologic milestone in this syndrome. In fact, one can say that sepsis is a disorder of the microcirculation being the first organ to fail during the progression of sepsis to the multiple organ dysfunction syndrome and death. It is now known that, despite adequate treatment of macro-hemodynamics, there is little correlation between the improvement in systemic hemodynamics and the correction of microcirculatory abnormalities, being the presence of these abnormalities more common among nonsurvivors than in survivors. The reason for this disparity between the macro and micro-hemodynamics is the presence of functional shunts in the microcirculation that "hide" the microcirculatory dysfunction from the systemic circulation. By not assessing the intrinsic events that occur in the microcirculation, conventional hemodynamic monitoring systems available in clinical practice fail to detect such abnormalities and response to treatments. This knowledge points to a great need in the diagnosis and treatment of sepsis: the diagnostic evaluation of the microcirculatory dysfunction. Fortunately, there are some diagnostic methods specifically targeted for the evaluation of the microcirculatory alterations. These methods can be divided into biomarkers, videomicroscopy techniques, techniques for assessment of the tissue oxygenation and techniques for evaluation of the microcirculation using the partial pressure of carbon dioxide. Aiming at a better understanding of the pathophysiological processes involved in the sepsis's microcirculatory dysfunction, and of the currently available diagnostic tools, some fundamental concepts are presented.

Keywords: Microcirculation; Sepsis; Monitoring, physiologic; Intensive care.

\section{Introdução}

Entre as diversas indicações de internação em unidade de terapia intensiva, a sepse ganha destaque pela sua alta incidência, morbidade, mortalidade e custos ao sistema de saúde. ${ }^{1,2}$

A disfunção da microcirculação é um elemento-chave na fisiopatologia da sepse. ${ }^{3} \mathrm{Na}$ tentativa de eliminar o agente agressor, ocorre a liberação de citocinas pró-inflamatórias gerando intensa resposta que agride a microcirculação. Estas alterações fisiopatológicas afetam quase todos os componentes celulares da microcirculação, incluindo as células endoteliais, as células musculares lisas, os leucócitos, os eritrócitos e as células do parênquima adjacente, levando à disfunção microcirculatória. Se não for corrigida, esta disfunção pode levar à hipóxia tecidual, que resulta na piora da disfunção microvascular, desencadeando uma cascata de mecanismos patogênicos que, por fim, conduzem à falência de órgãos. ${ }^{4}$

\section{A microcirculação}

A microcirculação é formada por vasos sanguíneos com diâmetro inferior a $100 \mu \mathrm{m}$, ou seja, as arteríolas, os capilares, as vênulas e os microlinfáticos; sendo o principal local de liberação de oxigênio do sangue para os tecidos. Os principais tipos celulares encontrados na microcirculação são as células endoteliais, que revestem o interior dos vasos, as células musculares lisas, presentes principalmente nas arteríolas, os eritrócitos, os leucócitos e as plaquetas. ${ }^{4}$

Em condições fisiológicas, a microcirculação pode ser entendida como um sistema que garante a adequação da oferta tecidual de oxigênio à demanda celular de oxigênio. Desta forma, na disfunção deste sistema, sobrevêm a má distribuição do fluxo sanguíneo e a hipóxia tecidual. As células endoteliais desempenham um papel central no sistema de controle do funcionamento da microcirculação por regular a trombose e fibrinólise microvascular, a adesão e migração leucocitária, o tônus arteriolar, a permeabilidade e o recrutamento capilar, todos 
determinantes do fluxo sanguíneo capilar e, por conseguinte, da oferta de oxigênio aos tecidos. ${ }^{3,5}$

\section{Fisiopatologia das alterações microcirculatórias na sepse}

Segundo Ince, a sepse é uma desordem da microcirculação. De fato, pode-se dizer que a microcirculação é o primeiro órgão a falhar na progressão da sepse para a síndrome de disfunção de múltiplos órgãos. ${ }^{6}$

Na sepse, a função dos principais tipos celulares encontrados na microcirculação está alterada (Figura 1), além disso, o sistema do óxido nítrico $(\mathrm{NO})$, um componente central na manutenção e autorregulação da homeostase e patência da microcirculação, está gravemente afetado. $^{3,4}$

Em estados fisiológicos, as células endoteliais regulam o fluxo microvascular através da liberação local de vasodilatadores, destacando-se em importância o NO. Este é um ativador da enzima guanilato ciclase, que é responsável pela produção de monofosfato cíclico de guanosina (cGMP), o mediador do relaxamento da musculatura lisa. Durante a sepse, a expressão heterogênea do óxido nítrico-sintase induzível (iNOS) em diferentes leitos vasculares orgânicos resulta no shunt patológico do fluxo, ou seja, no inadequado desvio do fluxo sanguíneo de unidades microvasculares em sofrimento via abertura de shunts arteriovenosos. Dessa forma, as áreas deficientes de iNOS, que têm menor vasodilatação induzida por NO, tornam-se hipoperfundidas. ${ }^{3,7} \mathrm{Na}$ sepse, além da alteração de expressão da iNOS, ocorre maior produção de radicais livres de oxigênio, que interferem com a produção de NO pela óxido nítrico-sintase endotelial (eNOS) e reagem com o NO já formado, reduzindo ainda mais a sua concentração. ${ }^{8}$

As células endoteliais da microcirculação perdem a sua capacidade de realizar a função reguladora durante o quadro séptico. Isto ocorre por causa de alterações em vias de transdução de sinais, da perda de comunicação eletrofisiológica e da perda do controle sobre a musculatura lisa arteriolar. ${ }^{4}$

Além de perderem a regulação pelas células endoteliais, as células musculares lisas das arteríolas perdem a sua sensibilidade adrenérgica e seu tônus, contribuindo ainda mais para as alterações perfusionais da microcirculação. ${ }^{9}$

Normalmente, o endotélio funciona como uma barreira antiadesiva para os componentes sanguíneos. Entretanto, na sepse, a presença de endotoxinas provoca um efeito pró-adesivo e pró-trombótico, promovendo a aderência de

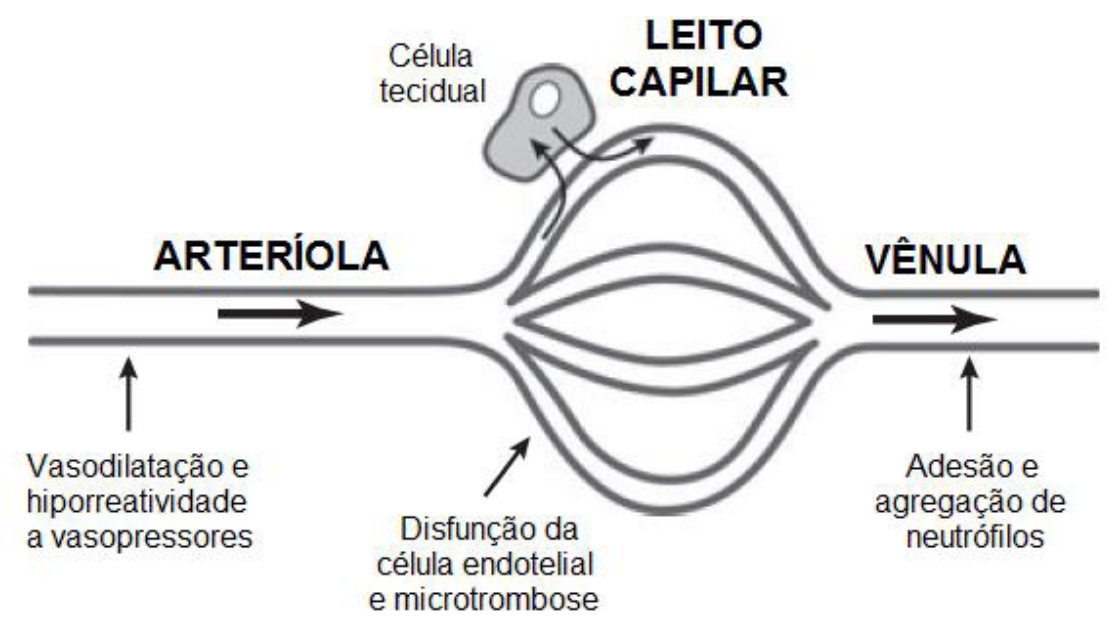

Figura 1. Alteração da função celular na microcirculação

Grande parte da fisiopatologia da sepse pode ser explicada por alterações que ocorrem nos elementos da microcirculação. Estas alterações incluem a perda da reatividade vasomotora, a lesão das células endoteliais, a ativação da coagulação e a aderência de leucócitos à parede do vaso e agregação, que prejudica o fluxo através da microcirculação. Fonte: Resuscitating the Microcirculation in Sepsis: the central role of nitric oxide, emerging concepts for novel therapies, and challenges for clinical trials, $2008 .^{3}$ 
eritrócitos, leucócitos e plaquetas ao endotélio vascular, o que compromete o fluxo capilar. ${ }^{8}$ Além disso, os eritrócitos tornam-se menos deformáveis e se agregam com maior facilidade durante a endotoxemia. ${ }^{10}$ Estas alterações, em conjunto com as alterações da coagulação, resultam na microtrombose vascular. ${ }^{4}$

Fisiologicamente, os eritrócitos também desempenham um papel importante na regulação do fluxo sanguíneo da microcirculação pela sua capacidade de liberar NO na presença de hipóxia, causando vasodilatação. Esta propriedade reguladora dos eritrócitos pode estar comprometida na sepse. ${ }^{11}$

Com relação aos leucócitos, a percentagem de neutrófilos ativados, com deformabilidade reduzida e agregabilidade aumentada devido a maior expressão de moléculas de adesão, aumenta durante a sepse. ${ }^{9}$ Os leucócitos ativados pela inflamação secundária à sepse geram espécies reativas de oxigênio que rompem diretamente as estruturas da microcirculação, as interações celulares e o equilíbrio da coagulação o que causa ainda maior adesão de leucócitos, plaquetas e eritrócitos ao endotélio vascular., ${ }^{42}$

A ação dos radicais livres de oxigênio e de outros mediadores inflamatórios prejudica a função de barreira da microcirculação, pois, ao interagirem com as junções entre as células e possivelmente com o glicocálice endotelial, levam ao edema tecidual e por conseguinte à piora do déficit de extração de oxigênio. ${ }^{13}$

Quando não corrigida, a disfunção da microcirculação leva à hipóxia celular e disfunção mitocondrial das células do parênquima, o que culmina na falência orgânica (Figura 2). ${ }^{4}$

Embora a disfunção microcirculatória possa ocorrer em graus variáveis em grande parte dos quadros que resultam em choque, os mecanismos de autorregulação da função microvascular são mais gravemente alterados durante a sepse, indicando a disfunção microcirculatória como um marco fisiopatológico na sepse. ${ }^{3}$

O papel central da microcirculação na oferta de oxigênio para as células dos tecidos determina a sua importância na manutenção da função dos órgãos. O funcionamento da microcirculação é fundamental para a sobrevivência e manutenção das células. A disfunção da microcirculação é caracterizada por heterogeneidade do fluxo sanguíneo, com alguns capilares sendo hipoperfundidos, enquanto outros têm fluxo de

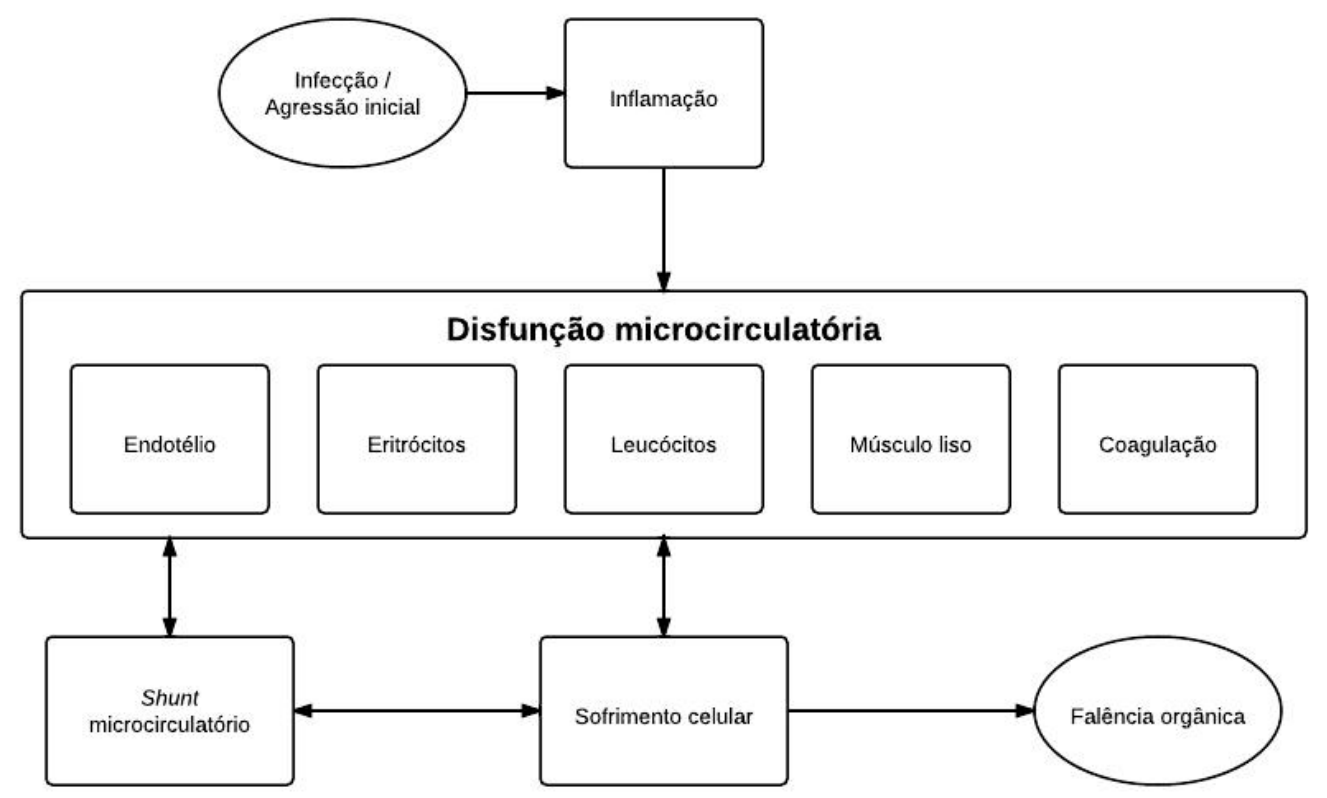

Figura 2. Esquema do mecanismo pelo qual a disfunção microcirculatória na sepse culmina na falência orgânica

Fonte: Adaptado de The microcirculation is the motor of sepsis, $2005 .{ }^{4}$ 
sangue normal ou, ainda, anormalmente alto. Algumas unidades microcirculatórias mais vulneráveis se tornam hipóxicas, determinando o déficit de extração de oxigênio associado à sepse. A falha em manter a função microvascular adequada resulta em hipoxemia ou anóxia do tecido, acúmulo de metabólitos e morte celular. ${ }^{4}$

As anormalidades microcirculatórias são características da síndrome séptica, sendo mais comuns entre os pacientes não sobreviventes que naqueles sobreviventes, pois a persistência das alterações microcirculatórias está associada ao desenvolvimento de falência orgânica e morte. ${ }^{14,15}$

Sabe-se atualmente que, a despeito de um tratamento adequado da macro-hemodinâmica, existe pouca correlação entre a melhora hemodinâmica sistêmica e a correção das anormalidades microcirculatórias. A razão para esta disparidade entre a macro e a micro-hemodinâmica é a presença de shunts funcionais na microcirculação que "escondem" a disfunção microcirculatória da circulação sistêmica. Em quadros patológicos (como a sepse), que determinam a desregulação da abertura destes shunts, a pressão parcial de oxigênio $\left(\mathrm{pO}_{2}\right)$ da microcirculação cai abaixo da venosa constituindo o " $\mathrm{pO}_{2}$ gap" e determinando sofrimento tecidual, apesar de parâmetros hemodinâmicos sistêmicos adequados. ${ }^{7}$ Este defeito distributivo associado com a sepse é caracterizado pela estagnação do fluxo sanguíneo em capilares, com redução da densidade de capilares funcionais, na presença de fluxo praticamente normal nos vasos maiores da microcirculação. ${ }^{4} \mathrm{~A}$ depressão do fluxo microvascular ocorre mais intensamente nos vasos menores, afetando menos os vasos maiores, o que explica em parte por que as variáveis hemodinâmicas sistêmicas não refletem de forma fidedigna as propriedades hemodinâmicas da microcirculação. ${ }^{6}$

Desta forma, por não avaliarem os eventos intrínsecos que ocorrem na microcirculação, os sistemas convencionais de monitorização hemodinâmica disponíveis na prática clínica falham em detectar estas alterações e a resposta aos tratamentos. Este conhecimento aponta para uma grande necessidade no diagnóstico e tratamento da sepse: a avaliação diagnóstica da disfunção da microcirculação.

\section{Aspectos diagnósticos}

O método ideal para a avaliação da microcirculação de pacientes críticos seria aquele que permitisse a realização desta avaliação à beira do leito e fosse capaz de avaliar o transporte local de oxigênio e as alterações na permeabilidade vascular, na resposta inflamatória e na coagulação. Finalmente, este método deveria permitir, de forma não invasiva, a visualização direta das alterações microvasculares, como a queda da densidade capilar funcional (número de capilares espontaneamente perfundidos por área analisada), dos diâmetros vasculares e das velocidades de fluxo. ${ }^{16}$ Infelizmente, não há um método único que seja capaz de monitorizar todas estas funções relacionadas à microcirculação. Além disso, qualquer que seja o método, deve-se ter em mente que apenas o leito microvascular para o qual o método esteja sendo utilizado é que será realmente avaliado naquele momento. Isto é particularmente importante na sepse, uma síndrome de caráter sistêmico na qual há grande heterogeneidade da microcirculação.

Os métodos atualmente disponíveis para uso clínico para a monitorização da perfusão microvascular podem ser divididos em biomarcadores, técnicas de videomicroscopia, técnicas para a avaliação da oxigenação tissular e técnicas para avaliação da microcirculação a partir da pressão parcial de gás carbônico.

\section{Biomarcadores}

A medida do lactato sérico arterial é muito utilizada na avaliação de pacientes críticos. Quando presente, a hiperlactatemia pode indicar hipoperfusão tecidual com aumento da produção de lactato por metabolismo anaeróbico. Quando ausente, não exclui a perfusão tissular inadequada, sendo um teste diagnóstico com sensibilidade e especificidade baixas. Ainda assim, a necessidade de observação de queda 
do lactato arterial em medidas seriadas faz parte das metas a serem alcançadas para o adequado tratamento da sepse grave/choque séptico, já havendo pesquisas publicadas mostrando correlação entre a queda do lactato e a melhora da perfusão microvascular. ${ }^{17,18}$

As moléculas de adesão (intercelular plasmática-1 [ICAM-1], celular vascular-1 [VCAM1], E-selectina e P-selectina) podem ser dosadas no soro dos pacientes sépticos e refletem a ativação endotelial (ou ruptura do endotélio) e a sua interação com leucócitos. Menos utilizado na prática clínica, o aumento sérico destas moléculas foi correlacionado com graves alterações na microcirculação de pacientes pediátricos com meningococcemia. ${ }^{19}$

\section{Avaliação direta da microcirculação (técnicas de videomicroscopia)}

Quanto à avaliação direta da microcirculação, a microscopia intravital, que historicamente necessitava de dissecções anatômicas para ser realizada em modelos animais, evoluiu com novas técnicas de microscopia minimamente invasiva que permitem, com o uso de instrumentos portáteis, a visualização direta da microcirculação em tecidos, utilizando a técnica de videocapilaroscopia ou a técnica de imagem espectral obtida através da polarização ortogonal (OPS - orthogonal polarization spectral imaging) ou ainda, mais recentemente, através da técnica denominada sidestream dark field (SDF), tornando possível a avaliação da microcirculação em seres humanos. ${ }^{12}$ A principal limitação de todos estes métodos é a dificuldade de seu uso de forma contínua, limitando as informações obtidas àquelas adquiridas no momento da realização do exame.

A videocapilaroscopia do leito ungueal foi o primeiro método utilizado à beira do leito e avalia os capilares localizados na junção entre a cutícula e a unha com o uso de um microscópio. Permite detectar anormalidades morfológicas, da densidade capilar e do fluxo microvascular em repouso e após testes de oclusão vascular (arterial e venosa). ${ }^{20} \mathrm{O}$ método não se popula- rizou na monitorização de pacientes sépticos, pois o leito ungueal é uma área muito sensível a mudanças de temperatura, vasoconstricção periférica e ao uso de agentes vasopressores, o que torna seu uso bastante restrito nesta população.

As técnicas de polarização ortogonal OPS e SDF são baseadas num mesmo princípio: o da utilização de luz polarizada com comprimento de onda de $530 \mathrm{~nm}$, que é absorvida pela hemoglobina eritrocitária, independente do seu estado de oxigenação, gerando imagens em tons de cinza. Desta forma, somente os vasos preenchidos por hemácias são visualizados. No OPS, a luz despolarizada fornece o contraste ao ser refletida pelas camadas mais profundas do tecido avaliado, gerando um fundo claro. No SDF, o contraste da imagem é dado por uma luz verde pulsátil periférica à óptica do aparelho. Infelizmente, por questões ligadas à técnica de obtenção de imagem, nenhum dos dois métodos permite a visualização direta das paredes dos vasos. A imagem captada é ampliada por meio óptico em 5 vezes e, por aumento digital, em mais 340 vezes com o OPS ou 380 vezes com o SDF. As técnicas de polarização ortogonal alcançam a profundidade de até 5 milímetros, permitindo a avaliação da microcirculação somente em tecidos cobertos por fina camada epitelial, sendo a mucosa sublingual o mais estudado e utilizado (Figura $3 \mathrm{~A}$ e B). A preferência por esta região se deve ao fato de sua origem embriológica ser a mesma que a do trato gastrointestinal. Desta forma, as alterações microcirculatórias encontradas na mucosa sublingual refletiriam de forma bastante satisfatória as alterações microcirculatórias esplâncnicas, consideradas fundamentais na origem e progressão da síndrome séptica até a falência de múltiplos órgãos. ${ }^{21}$ Nas imagens são analisadas a densidade capilar funcional, o tipo de fluxo predominante e o grau de heterogeneidade. ${ }^{22}$ Os principais limitantes do uso desta tecnologia são a necessidade de equipamento específico, de treinamento do operador (a fim de evitar artefatos de pressão e movimento) e a possibilidade de sangramento da mucosa analisada. 


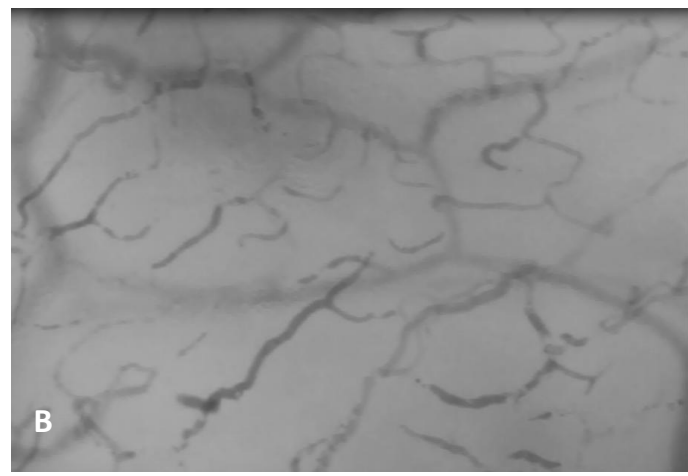

Figura 3. Imagens obtidas por SDF mostrando a microcirculação da mucosa sublingual em dois momentos distintos num mesmo paciente: A. ausência de choque; B. em vigência de choque séptico

Fonte: Imagens cedidas pela Drạ. Daniella Mancino da Luz Caixeta.

Embora a avaliação direta da microcirculação possa fornecer informações sobre a perfusão tecidual que não estão disponíveis a partir dos parâmetros macrocirculatórios, a imagem por si só não fornece informações sobre a real oferta e utilização de oxigênio pelas células. Desta forma, além da disfunção da microcirculação, alguns mecanismos a nível celular, tais como a disfunção mitocondrial e a apoptose, também são considerados fatores centrais no desenvolvimento da morte celular e falência orgânica associadas à sepse. No entanto, estas anormalidades são uma resposta adaptativa tardia que pode ser precedida ou disparada pela insuficiência microcirculatória, reforçando a importância da sua avaliação. ${ }^{3,23}$ Como forma de complementar as informações oferecidas pela avaliação direta da microcirculação, as anormalidades de oferta e consumo de oxigênio, a nível celular, podem ser indiretamente avaliadas pela tonometria gástrica, pela dosagem sanguínea ou tecidual do lactato, $\mathrm{pH}$ e excesso de base ou pela monitorização da saturação venosa mista ou central de oxigênio ou do gradiente venoarterial da pressão parcial de dióxido de carbono (delta $\left.\mathrm{CO}_{2}\right){ }^{24,25}$

\section{Avaliação da oxigenação tissular}

O principal objetivo do tratamento da sepse é adequar o transporte e a oferta tissular de oxigênio à condição patológica de consumo. Desta forma, a monitorização direta da alteração provocada pela sepse e da resposta ao tratamento envolveria a avaliação de todos os componentes envolvidos na relação oferta/ consumo de oxigênio, o que, na prática, não é possível com a tecnologia disponível atualmente. No entanto, métodos de avaliação indireta podem ser rotineiramente utilizados.

$O$ consumo de oxigênio pode ser indiretamente avaliado por métodos como a monitorização da saturação tissular de oxigênio (pela técnica de espectroscopia óptica na região do infravermelho próximo (NIRS - Near-infrared spectroscopy) e da saturação venosa de oxigênio.

A técnica de NIRS utiliza ondas eletromagnéticas na região espectral do infravermelho próximo aproveitando mudanças das propriedades ópticas dos tecidos para medir os cromóforos oxi e desoxi-hemoglobina, mioglobina e citocromo aa3 oxidado. As medidas de oxi e desoxi-hemoglobina são utilizadas para calcular a saturação do oxigênio tissular $\left(\mathrm{StO}_{2}\right)$; já a medida de citocromo aa3 oxidado permite a avaliação da tensão de oxigênio mitocondrial. Como o comprimento de onda utilizado, variando aproximadamente entre 650 e $900 \mathrm{~nm}$, atravessa a pele, o tecido subcutâneo, o músculo esquelético e o tecido ósseo, a NIRS é capaz de penetrar em órgãos por até alguns centímetros, o que permite a monitorização da $\mathrm{StO}_{2}$ órgão-específica. Os órgãos mais utilizados para a monitorização pela NIRS são o cérebro e o músculo. A saturação de oxigênio tissular pode ser avaliada em repouso e após testes de oclusão venosa e arterial. $\mathrm{O}$ valor da $\mathrm{StO}_{2}$ em repouso medida pela NIRS reflete principalmente o com- 
partimento venoso. Já os valores obtidos após testes de oclusão vascular permitem os cálculos do consumo muscular de oxigênio $\left(\mathrm{mVO}_{2}\right)$, do fluxo sanguíneo periférico e das velocidades da desoxigenação e reoxigenação. As principais limitações do método são a presença de panículo adiposo espesso e/ou edema no local de aplicação do sensor, a presença de baixa temperatura e o uso de drogas vasoativas. ${ }^{26}$

A avaliação do consumo de oxigênio através da medição da saturação de oxigênio em sangue venoso obtido por cateter venoso central (saturação venosa central de oxigênio) é muito utilizada na prática clínica para guiar a ressuscitação de pacientes sépticos. Seu valor reflete o balanço entre o transporte e o consumo global de oxigênio, mas não mostra correlação direta com a disfunção microvascular nestes pacientes. ${ }^{21}$

O transporte de oxigênio também pode ser indiretamente estimado. Para tanto, a avaliação transcutânea da $\mathrm{pO}_{2}$ é um método comumente utilizado. Inicialmente criado para ser correlacionado com a $\mathrm{pO}_{2}$ arterial, evitando coletas seriadas de amostras de sangue arterial para gasometrias, o método utiliza o sensor do tipo Stow-Severinghaus acoplado à pele. Como nos pacientes sépticos, especialmente naqueles com choque, não há correlação entre a oxigenação arterial e a oxigenação da pele, a $\mathrm{pO}_{2}$ transcutânea pode ser interpretada como uma estimativa da perfusão da pele (ou do transporte de oxigênio). Além do dado bruto, pode-se avaliar também a tendência da oxigenação transcutânea para o diagnóstico de choque e a otimização da terapêutica. As principais limitações do método são a presença de lesões de pele que impeçam a instalação dos eletrodos, de edema ou de panículo adiposo espesso e o uso de drogas vasoativas. ${ }^{27}$

Avaliação da microcirculação a partir da pressão parcial de gás carbônico

A pCO $\mathrm{pC}_{2}$ tissular representa o balanço entre o fluxo local e a produção de gás carbônico. A medição transcutânea da $\mathrm{pCO}_{2}$ pode ser obtida a partir do mesmo monitor e sensor que medem a $\mathrm{pO}_{2}$ transcutânea. A interpretação dos dados é semelhante: nos pacientes com sepse, especialmente naqueles com choque, se a $\mathrm{pCO}_{2}$ transcutânea está baixa na presença de normocapnia, a perfusão local deve estar prejudicada, pois a redução da oferta de oxigênio termina reduzindo a produção de gás carbônico. Além do dado bruto, a tendência também deve ser avaliada. As limitações do método são as mesmas da monitorização transcutânea da $\mathrm{pO}_{2}$, além da presença de hipercapnia. ${ }^{28}$

A tonometria gástrica foi um método muito pesquisado e utilizado no passado. É uma técnica pouco invasiva que mede a $\mathrm{pCO}_{2} \mathrm{da}$ mucosa gástrica, monitorizando a adequação do balanço entre a oferta e o consumo de oxigênio nesta mucosa sendo, portanto, órgão-específica. Em pacientes sépticos não há correlação entre a $\mathrm{pCO}_{2}$ gástrica e a perfusão esplâncnica global, limitando as informações oferecidas pelo método, embora haja correlação com a ocorrência de mudanças na perfusão quando a $\mathrm{pCO}_{2}$ gástrica é avaliada de forma seriada. ${ }^{29} \mathrm{~A}$ principal limitação da técnica, responsável pela restrição do seu uso, é a interferência na medida causada pela presença de alimentos no estômago e pelo refluxo duodenal.

\section{Conclusão}

A disfunção da microcirculação tem papel central na gênese e manutenção da síndrome séptica, sendo um marco fisiopatológico desta síndrome.

Infelizmente, o diagnóstico das alterações microcirculatórias e a adoção de medidas terapêuticas direcionadas ao tratamento destas alterações ainda não fazem parte do manejo da maioria dos pacientes sépticos, seja pela falta de tecnologia adequada, seja pela falta de conhecimento e/ou treinamento dos profissionais que assistem a estes pacientes.

Uma mudança de paradigma, concentrando as atenções, diagnósticas e terapêuticas, não mais na macro-hemodinâmica e sim na microcirculação pode contribuir para a redução da ainda alta morbimortalidade associada à sepse. 


\section{Referências}

1. Sales Júnior JAL, David CM, Hatum R, et al. Sepse Brasil: Estudo Epidemiológico da Sepse em Unidades de terapia intensiva Brasileiras. Rev Bras Ter Intensiva. 2006;18(1):9-17.

2. Martin GS, Mannino DM, Eaton S, Moss M. The Epidemiology of Sepsis in the United States from 1979 through 2000. N Engl J Med. 2003 Apr 17;348(16):1546-54.

3. Trzeciak S, Cinel I, Dellinger R, Shapiro NI, Arnold RC, Parrillo JE, et al. Resuscitating the Microcirculation in Sepsis: The Central Role of Nitric Oxide, Emerging Concepts for Novel Therapies, and Challenges for Clinical Trials. Acad Emerg Med. 2008;15(5):399-413. http:// dx.doi.org/10.1111/j.1553-2712.2008.00109.x.

4. Ince $\mathrm{C}$. The microcirculation is the motor of sepsis. Crit Care. 2005;9(Suppl 4):S13-S19.

5. Aird WC. Endothelium as an organ system. Crit Care Med. 2004;32(Suppl 5):S271-9.

6. Ince C. The Microcirculation Unveiled. Am J Respir Crit Care Med. 2002;166(1):1-2.

7. Ince C, Sinaasappel M. Microcirculatory oxygenation and shunting in sepsis and shock. Crit Care Med. 1999;27(7):1369-77.

8. Cepinskas G, Wilson JX. Inflammatory Response in Microvascular Endothelium in Sepsis: Role of Oxidants. J Clin Biochem Nutr. 2008;42(3):175-84. http://dx.doi.org/10.3164/ jcbn.2008026.

9. Tyagi A, Sethi AK, Girotra G, Mohta M. The Microcirculation in Sepsis. Indian J Anaesth. 2009;53(3):281-93.

10. Eichelbrönner O, Sielenkämper A, Cepinskas G, Sibbald WJ, Chin-Yee IH. Endotoxin promotes adhesion of human erythrocytes to human vascular endothelial cells under conditions of flow. Crit Care Med. 2000;28(6):1865-70.

11. Singel DJ, Stamler JS. Chemical Physiology of Blood Flow Regulation by Red Blood Cells: The Role of Nitric Oxide and S-Nitrosohemoglobin. Annu Rev Physiol. 2005;67:99-145.

12. Penna GL, Salgado DR, Japiassú AM, Kalichsztein M, Nobre GF, Villela N, et al. Avaliação da microcirculação: uma nova arma no manejo da sepse? Rev Bras Ter Intensiva. 2011;23(3):352-7.

13. van den Berg BM, Vink H, Spaan JAE. The Endothelial Glycocalyx Protects Against Myocardial Edema. Circ Res. 2003; 92(6):592-4.

14. Trzeciak S, McCoy JV, Dellinger R, Arnold RC, Rizzuto M, Abate NL, et al. Early increases in microcirculatory perfusion during protocol-directed resuscitation are associated with reduced multi-organ failure at $24 \mathrm{~h}$ in patients with sepsis. Intensive Care Med. 2008; 34(12):2210-17. http://dx.doi. org/10.1007/s00134-008-1193-6
15. Sakr Y, Dubois MJ, De Backer D, Creteur J, Vincent JL. Persistent microcirculatory alterations are associated with organ failure and death in patients with septic shock. Crit Care Med. 2004;32(9):1825-31.

16. De Backer D, Ospina-Tascon G, Salgado D, Favory R, Creteur J, Vincent JL. Monitoring the microcirculation in the critically ill patient: current methods and future approaches. Intensive Care Med. 2010;36(11):1813-25. http://dx.doi.org/10.1007/ s00134-010-2005-3.

17. Arnold R, Shapiro N, Jones A, Schorr C, Pope J, Casner E, et al. Multi-center study of early lactate clearance as a determinant of survival in patients with presumed sepsis. Shock. 2009;32(1):35-9.

18. Ospina-Tascon G, Neves A, Occhipinti G, Donadello K, Büchele G, Simion D, et al. Effects of fluids on microvascular perfusion in patients with severe sepsis. Intensive Care Med. 2010;36(6):949-55. http://dx.doi. org/10.1007/s00134-010-1843-3.

19. Paize F, Sarginson R, Makwana N, Baines PB, Thomson AP, Sinha I, et al. Changes in the sublingual microcirculation and endothelial adhesion molecules during the course of meningococcal disease treated in the paediatric intensive care unit. Intensive Care Med. 2012;38(5):863-71. http://dx.doi. org/10.1007/s00134-012-2476-5.

20. Dancour M, Vaz J, Bottino D, Bouskela E Nailfold videocapillaroscopy in patients with systemic lupus erythematosus. Rheumatol Int. 2006;26(7): 633-7.

21. De Backer D, Creteur J, Preiser J, Dubois MJ, Vincent JL. Microvascular blood flow is altered in patients with sepsis. Am J Respir Crit Care Med. 2002;166(1):98-104.

22. De Backer D, Hollenberg S, Boerma C, Goedhart P, Büchele G, Ospina-Tascon G, et al. How to evaluate the microcirculation: report of a round table conference. Crit Care. 2007;11(5):R101.

23. Singer M, De Santis V, Vitale D, Jeffcoate W. Multiorgan failure is an adaptive, endocrine-mediated, metabolic response to overwhelming systemic inflammation. Lancet. 2004;364(9433):545-8.

24. Huang YCT. Monitoring Oxygen Delivery in the Critically Ill. Chest. 2005;128(5 Suppl 2):554S-560S.

25. Réa-Neto Á, Rezende E, Mendes C, et al. Consenso brasileiro de monitorização e suporte hemodinâmico - Parte IV: monitorização da perfusão tecidual. Rev Bras Ter Intensiva. 2006;18(2):154-60.

26. Lima A, Bakker J. Near-infrared spectroscopy for monitoring peripheral tissue perfusion in critically ill patients. Rev Bras Ter Intensiva. 2011;23(3):341-51.

27. Barker S, Tremper KK. Transcutaneous 
Revista HUPE, Rio de Janeiro, 2013;12(3):21-30

oxygen tension: A physiological variable for monitoring oxygenation. J Clin Monit. 1985;1(2):130-4.

28. Rodriguez P, Lellouche F, Aboab J, Buisson $\mathrm{CB}$, Brochard L. Transcutaneous arterial carbon dioxide pressure monitoring in critically ill adult patients. Intensive Care Med. 2006;32(2):309-12.

29. Creteur J, De Backer D, Vincent JL. Does gastric tonometry monitor splanchnic perfusion? Crit Care Med. 1999;27(11):2480-84. 